PROCEEDINGS OF THE

AMERICAN MATHEMATICAL SOCIETY

Volume 124, Number 12, December 1996, Pages 3627-3636

S 0002-9939(96)03653-2

\title{
A SUM PACKING PROBLEM OF ERDŐS AND THE CONWAY-GUY SEQUENCE
}

\author{
TOM BOHMAN
}

(Communicated by Jeffry N. Kahn)

\begin{abstract}
A set $S$ of positive integers has distinct subset sums if the set $\left\{\sum_{x \in X} x: X \subset S\right\}$ has $2^{|S|}$ distinct elements. Let

$f(n)=\min \{\max S:|S|=n$ and $S$ has distinct subset sums $\}$.

In 1931 Paul Erdős conjectured that $f(n) \geq c 2^{n}$ for some constant $c$. In 1967 John Conway and Richard Guy constructed an interesting sequence of sets of integers. They conjectured that these sets have distinct subset sums and that they are close to the best possible (with respect to largest element). We prove that sets from this sequence have distinct subset sums. We also present some variations of this construction that give microscopic improvements in the best known upper bound on $f(n)$.
\end{abstract}

\section{INTRODUCTION}

A set $S$ of positive integers has distinct subset sums if the set $\left\{\sum_{x \in X} x: X \subset S\right\}$ has $2^{|S|}$ distinct elements. Let

$$
f(n)=\min \{\max S:|S|=n \text { and } S \text { has distinct subset sums }\} .
$$

By taking the set $S$ to be the first $n$ powers of 2 we see that $f(n) \leq 2^{n-1}$. Some small examples (the sets $\{3,5,6,7\}$ and $\{6,9,11,12,13\}$ for example) suggest that $f(n)$ could be much smaller than $2^{n-1}$. In 1931 Erdös conjectured that this is not the case [E1]. In particular, he conjectured that $f(n) \geq c 2^{n}$ for some constant $c$, and he now offers $\$ 500$ for settling this conjecture [E1], [G1], [G2].

In 1955 Erdős and Moser proved that $f(n) \geq 2^{n} /(10 \sqrt{n})$ [E2] (for a proof using the second moment method see [AS, p. 47]). This remains, up to the constant, the best known lower bound.

The first nontrivial upper bound on $f(n)$ was $2^{n-2}$ (for $n$ sufficiently large). This bound was achieved by Conway and Guy when, in 1967, they showed that the first 40 sets from the Conway-Guy sequence (defined below) have distinct subset sums [CG], [G1], [G2]. The $21^{\text {st }}$ set in this sequence has largest element slightly smaller than $2^{19}$. This gives the bound of $2^{n-2}$ for all $n>21$ by the following construction: given a set $S$ with $n$ elements and largest element $m$ we construct a set $S^{\prime}$ with $n+1$ elements and largest element $2 m$ by doubling every element in $S$ and introducing an odd number.

Received by the editors June 6, 1995

1991 Mathematics Subject Classification. Primary 11P99; Secondary 05 D10.

(C)1996 American Mathematical Society 
Let $S_{1}, S_{2}, S_{3}, \ldots$ be the sets from the Conway-Guy sequence. $\max S_{n} / 2^{n}$ is strictly decreasing for $n>3$ and converges to approximately .23513. So proving that all sets in the Conway-Guy sequence have distinct subset sums yields a very small improvement in the upper bound. Furthermore, the Conway-Guy bound has been surpassed by a set of 67 integers found by Lunnon which gives $f(n)<.2246\left(2^{n}\right)$ [L]. The best known upper bound on $f(n)$ now stands at approximately $.22002\left(2^{n}\right)$. The construction which achieves the new bound is an improvement on Lunnon's construction and is given in section 4 .

In the remainder of this section we give an outline of the technique we use to show that sets have distinct subset sums and apply this technique to a simple example. Section 2 contains the proof that the sets from the Conway-Guy sequence have distinct subset sums. Sections 3 and 4 are discussions of two interesting generalizations of this construction. Section 5 consists mostly of a conjecture that was motivated by this work.

We begin by considering a condition which is equivalent to the distinct subset sums condition. Note that $S$ fails to have distinct subset sums if and only if there are disjoint $I, J \subset S$ with $\sum I=\sum J$ (for a set of integers $X$ we write $\sum X$ for $\left.\sum_{x \in X} x\right)$. This is equivalent to a condition on the differences between the integers in our set. Let $S=\left\{a_{1}<a_{2}<\ldots<a_{n}\right\}$ be an arbitrary set of positive integers. We form the difference vector of $S: \mathbf{d}_{S}=\left(a_{1}, a_{2}-a_{1}, \ldots, a_{n}-a_{n-1}\right)$. The condition on the differences is determined by changing the order of summation in the distinct sums condition on subsets of $S$. To state the condition precisely we need the following definition.

Definition. An $n$-dimensional vector $\mathbf{v}$ with integer-valued components will be called smooth if

$$
|\mathbf{v}(n)| \leq 1 \quad \text { and } \quad|\mathbf{v}(i)-\mathbf{v}(i+1)| \leq 1 \quad \text { for } \quad i=1, \ldots, n-1 .
$$

Lemma 1. Let $S$ be a set of $n$ positive integers. There exist $I, J \subset S$ such that $I \cap J=\emptyset$ and $\sum I=\sum J \Longleftrightarrow$ there exists a nonzero, smooth, $n$-dimensional vector $\boldsymbol{v}$ such that $\mathbf{v} \cdot \mathbf{d}_{S}=0$.

Proof. $(\Rightarrow)$ Let $I=\left\{y_{1}, y_{2}, \ldots, y_{k}\right\}, J=\left\{z_{1}, z_{2}, \ldots, z_{l}\right\}$, and suppose $\sum I=\sum J$, i.e.

$$
\sum_{i=1}^{k} y_{i}-\sum_{i=1}^{l} z_{i}=0
$$

For appropriate choices of $\alpha_{i}$ and $\beta_{i}$, we have:

$$
y_{i}=\sum_{j=1}^{\alpha_{i}} \mathbf{d}_{S}(j), \quad z_{i}=\sum_{j=1}^{\beta_{i}} \mathbf{d}_{S}(j) .
$$

Notice that the $\alpha_{i}$ 's and $\beta_{i}$ 's are all distinct. We can write:

$$
\sum_{i=1}^{k} \sum_{j=1}^{\alpha_{i}} \mathbf{d}_{S}(j)-\sum_{i=1}^{l} \sum_{j=1}^{\beta_{i}} \mathbf{d}_{S}(j)=0 .
$$

In order to reverse the order of summation we must count how many times each $\mathbf{d}_{S}(j)$ appears in this summation. This is achieved by setting:

$$
\mathbf{v}(j)=\left|\left\{i: 1 \leq j \leq \alpha_{i}\right\}\right|-\left|\left\{i: 1 \leq j \leq \beta_{i}\right\}\right| .
$$


Then $\mathbf{v} \cdot \mathbf{d}_{S}$ is the sum on the left hand side of the above equation, and $\mathbf{v}$ is smooth by the distinctness of the $\alpha_{i}$ 's and $\beta_{i}$ 's .

$(\Leftarrow)$ Any smooth vector can be written as the sum of \pm 1 multiples of characteristic vectors of intervals that have left endpoints at 1 and distinct right endpoints. By reversing the above process, we can see how such a set of characteristic vectors gives us sets $I$ and $J$.

So to show that $S$ has distinct subset sums we must show that $\mathbf{v} \cdot \mathbf{d}_{S} \neq 0$ for all nonzero smooth vectors $\mathbf{v}$. Let $\mathbf{v}$ be a nonzero smooth vector. For the sets of integers we consider there is a natural way to construct a sequence $\mathbf{w}_{n}, \mathbf{w}_{n-1}, \ldots, \mathbf{w}_{2}$ of approximations of $\mathbf{v}$ that have the following two properties:

1. $\mathbf{w}_{i}$ agrees with $\mathbf{v}$ in the $n-i+1$ coordinates where $\mathbf{d}_{S}$ is greatest.

2. $\mathbf{w}_{i} \cdot \mathbf{d}_{S}=0$ for all $i$.

Once we establish what these approximations are we will be able to show that they satisfy

3. If $\mathbf{w}_{i}$ is nonzero then $\mathbf{w}_{i}$ is not smooth.

This will imply $\mathbf{v} \cdot \mathbf{d}_{S} \neq 0$ because $\mathbf{w}_{2}$ will agree with $\mathbf{v}$ in exactly $n-1$ coordinates and $\mathbf{w}_{2} \cdot \mathbf{d}_{S}$ will equal zero. The proofs in this paper follow this general outline. When proving that sets in the Conway-Guy sequence have distinct subset sums we will refine this approach by eliminating special classes of $\mathbf{v}$ 's from consideration in the course of the argument. We now consider a simple example.

This example is a generalization of the powers of 2 . Note that if $S=\{1,2,4, \ldots$, $\left.2^{n-1}\right\}$ then $\mathbf{d}_{S}=\left(1,1,2, \ldots, 2^{n-2}\right)$. For our example we will permute these differences to get other sets of integers that have distinct subset sums. Let $\sigma$ be a permutation on $n$ elements that satisfies the property:

$$
\forall i>1 \quad \exists j<i \text { such that }|\sigma(j)-\sigma(i)|=1 .
$$

Then set $\mathbf{d}_{S^{\prime}}(\sigma(1))=1$ and $\mathbf{d}_{S^{\prime}}(\sigma(i))=2^{i-2}$ for $i>1$. We will now show that the set $S^{\prime}$ that corresponds to this difference vector has distinct subset sums.

Let $\mathbf{v}$ be a smooth vector. We will construct a sequence $\mathbf{w}_{n}, \mathbf{w}_{n-1}, \ldots, \mathbf{w}_{2}$ as indicated above. For $i=2, \ldots, n$ set the vector

$$
\mathbf{x}_{\sigma(i)}(j)=\left\{\begin{aligned}
1 & \text { if } \sigma(i)=j \\
-1 & \text { if } j=\sigma(k) \text { for some } k<i \\
0 & \text { otherwise. }
\end{aligned}\right.
$$

The $\mathbf{w}_{i}$ 's will be linear combinations of the $\mathbf{x}_{i}$ 's. It is easy to see by the definition of $\mathbf{d}_{S^{\prime}}$ that $\mathbf{d}_{S^{\prime}} \cdot \mathbf{x}_{i}=0$ for $i \in\{2, \ldots, n\}$. It follows that the $\mathbf{w}_{i}$ 's will have property 2 . To start, set $\mathbf{w}_{n}=\mathbf{v}(\sigma(n)) \mathbf{x}_{\sigma(n)}$. This vector agrees with $\mathbf{v}$ in the $\sigma(n)$ coordinate, so it satisfies property 1 above. Now, iteratively apply

$$
\mathbf{w}_{i}=\mathbf{w}_{i+1}+\left(\mathbf{v}(\sigma(i))-\mathbf{w}_{i+1}(\sigma(i))\right) \mathbf{x}_{\sigma(i)} \quad \text { for } \quad 2 \leq i \leq n-1
$$

to get the rest of the $\mathbf{w}_{i}$ 's. Clearly, $\mathbf{w}_{i}$ agrees with $\mathbf{v}$ in the $\sigma(i)$ coordinate. Since $\mathbf{x}_{\sigma(j)}(\sigma(i))=0$ if $i>j$, the agreement between $\mathbf{v}$ and our approximation in the $\sigma(i)$ coordinate will be preserved in all approximations after $\mathbf{w}_{i}$ (i.e. $\mathbf{w}_{j}$ where $j<i$ ). Thus, the sequence satisfies property 1.

All we have to do to finish the proof is show that the nonzero $\mathbf{w}_{i}$ 's are not smooth. Suppose $\mathbf{w}_{i} \neq \mathbf{0}$. Let $k$ be the smallest integer greater than or equal to $i$ for which $M:=\mathbf{v}(\sigma(k))-\mathbf{w}_{k+1}(\sigma(k)) \neq 0$. This is the last place where there was a change in our approximation, and so $\mathbf{w}_{i}=\mathbf{w}_{k}$. By (1) there exists an index $j$ smaller 
than $k$ for which $|\sigma(j)-\sigma(k)|=1$. Then with $L:=\mathbf{w}_{k+1}(\sigma(j))=\mathbf{w}_{k+1}(\sigma(k))$ we have $\mathbf{w}_{k}(\sigma(k))=L+M$ while $\mathbf{w}_{k}(\sigma(j))=L-M$. This violates the smoothness condition between the indices $\sigma(j)$ and $\sigma(k)$.

\section{The Conway-Guy sequence}

The Conway-Guy sequence is a sequence of sets of integers constructed by the following three step procedure:

1. Set $\mathbf{b}(n)=[\sqrt{2(n-1)}]$ ([ ] is the nearest integer function) for $n \geq 1$.

2. We use a recursive rule to construct a sequence $\{\mathbf{d}(n)\}$ of 'differences'. We can think of $\{\mathbf{b}(n)\}$ as a 'Fibonacci guide' for the construction of $\{\mathbf{d}(n)\}$. In particular, $\mathbf{d}(1)=1$ and

$$
\mathbf{d}(i)=\sum_{j=i-\mathbf{b}(i)}^{i-1} \mathbf{d}(j) \text { for } i>1 .
$$

3. The sets in the Conway-Guy sequence are

$$
S_{i}=\left\{\sum_{j=k}^{i} \mathbf{d}(j): k=1, \ldots, i\right\} .
$$

Here are the first few terms in these sequences:

$$
\begin{aligned}
& \{\mathbf{b}(n)\} \quad: \quad 0, \quad 1, \quad 2, \quad 2, \quad 3, \quad 3, \quad 3, \quad 4, \quad 4, \quad 4, \quad 4, \quad 5, \quad \ldots, \\
& \{\mathbf{d}(n)\} \quad: \quad 1, \quad 1, \quad 2, \quad 3, \quad 6, \quad 11, \quad 20, \quad 40, \quad 77, \quad 148, \quad 285, \quad 570, \ldots \text {, } \\
& S_{1}=\{1\}, \quad S_{2}=\{2,1\}, \quad S_{3}=\{4,3,2\}, \quad S_{4}=\{7,6,5,3\}, \\
& \ldots, \quad S_{8}=\{84,83,82,80,77,71,60,40\} \text {, }
\end{aligned}
$$

The important thing to note about the sequence $\mathbf{b}$ is that each positive integer $i$ appears in the sequence exactly $i$ times. One consequence of this (and the thing that will be crucial in what follows) is that

$$
\mathbf{b}(i-\mathbf{b}(i))<\mathbf{b}(i) \text { for } i \geq 2 \text {. }
$$

We can now state the central result:

Theorem 1. For any positive integer $i$ the set $S_{i}$ has distinct subset sums.

We should also note that traditionally the Conway-Guy sequence is not constructed in this way. In place of the sequence $\{\mathbf{d}(n)\}$ the original construction makes use of a sequence $\{\mathbf{u}(n)\}$ defined by $\mathbf{u}(0)=0, \mathbf{u}(1)=1$ and $\mathbf{u}(i+1)=$ $2 \mathbf{u}(i)-\mathbf{u}(i-\mathbf{b}(i+1))$. Then, the sets of integers are obtained by taking differences of elements from this sequence. To be precise: $S_{n}=\{\mathbf{u}(n+1)-\mathbf{u}(i): i=1, \ldots, n\}$. Since $\mathbf{u}(i+1)-\mathbf{u}(i)=\mathbf{d}(i+1)$, these two constructions yield the same sets of integers. The advantage of the original construction is that it gives the sequence of greatest elements of the sets in the Conway-Guy sequence. We start with the alternate construction because it contains the difference vectors for the Conway-Guy sequence that we will need in the proof.

The difference vector for $S_{n}$ is $(\mathbf{d}(n), \mathbf{d}(n-1), \ldots, \mathbf{d}(1))$. We will think of $\mathbf{d}$ as an infinite dimensional vector. If $\mathbf{v}$ is a vector of dimension $n$, we will write $\mathbf{v} \cdot \mathbf{d}$ for the dot product of $\mathbf{v}$ with the first $n$ components of $\mathbf{d}$. This is the dot product of a smooth vector with the difference vector of $S_{n}$ if $|\mathbf{v}(1)| \leq 1$ and $|\mathbf{v}(i)-\mathbf{v}(i+1)| \leq 1$ for $1 \leq i \leq n-1$. So for ease of notation, we will reverse the order in the definition 
of a smooth vector. From now on, a smooth vector will be one for which $|\mathbf{v}(1)| \leq 1$ and $|\mathbf{v}(i)-\mathbf{v}(i+1)| \leq 1$ for $1 \leq i \leq n-1$. Hence, showing that $\mathbf{v} \cdot \mathbf{d} \neq 0$ for any finite dimensional smooth vector $\mathbf{v}$ is equivalent to proving Theorem 1 . Preparatory to proving Theorem 1, we establish some simple lemmas about $\mathbf{d}$.

Lemma 2. $\mathbf{d}(n+2) \geq \sum_{i=1}^{n} \mathbf{d}(i)$.

Proof. This is true for $n=1$, and for larger $n$ it follows by induction:

$$
\mathbf{d}(n+2) \geq \mathbf{d}(n+1)+\mathbf{d}(n) \geq \sum_{i=1}^{n-1} \mathbf{d}(i)+\mathbf{d}(n)=\sum_{i=1}^{n} \mathbf{d}(i) .
$$

Lemma 3. If $\boldsymbol{v}$ is a $n$-dimensional smooth vector with $|\mathbf{v}(n)| \leq 1$, then $\mathbf{v} \cdot \mathbf{d}<$ $\mathbf{d}(n+2)+\mathbf{d}(n+3)$.

Proof. We go by induction and use Lemma 2. Without loss of generality $\mathbf{v}(n) \geq$ 0 . Define the vector $\mathbf{z}$ by $\mathbf{z}(i)=\max \{\mathbf{v}(i)-1,0\}$. We can apply the inductive hypothesis to the first $n-1$ components of $\mathbf{z}$ since $\mathbf{z}$ is smooth and $\mathbf{z}(n)=0$. Letting $\mathbf{1}$ be the $n$-dimensional vector of 1 's, i.e. $\mathbf{1}=(1,1, \ldots 1)$, we have:

$$
\begin{aligned}
\mathbf{v} \cdot \mathbf{d} & =(\mathbf{v}-\mathbf{1}) \cdot \mathbf{d}+\mathbf{1} \cdot \mathbf{d} \\
& \leq \mathbf{z} \cdot \mathbf{d}+\mathbf{1} \cdot \mathbf{d} \\
& <(\mathbf{d}(n+1)+\mathbf{d}(n+2))+\mathbf{d}(n+2) \\
& \leq \mathbf{d}(n+3)+\mathbf{d}(n+2) .
\end{aligned}
$$

Lemma 4. If $\boldsymbol{v}$ is a smooth $n$-dimensional vector with $|\mathbf{v}(n)| \geq 2$, then $\mathbf{v} \cdot \mathbf{d} \neq 0$.

Proof. Without loss of generality, we can assume that $\mathbf{v}(n) \geq 2$. It follows from the smoothness of $\mathbf{v}$ that $\mathbf{v}(n-1) \geq 1$ and $\mathbf{v}(n-2) \geq 0$. So

$$
\mathbf{v} \cdot \mathbf{d}>\mathbf{v}^{\prime} \cdot \mathbf{d}+\mathbf{d}(n-1)+\mathbf{d}(n) \geq \mathbf{v}^{\prime \prime} \cdot \mathbf{d}+\mathbf{d}(n-1)+\mathbf{d}(n)
$$

where $\mathbf{v}^{\prime}$ is the restriction of $\mathbf{v}$ to its first $n-2$ components, and $\mathbf{v}^{\prime \prime}$ is the $(n-2)-$ dimensional vector defined by $\mathbf{v}^{\prime \prime}(i)=\min \{\mathbf{v}(i), 0\}$. Note that $\mathbf{v}^{\prime \prime}(n-2)=0$ and $\mathbf{v}^{\prime \prime}$ is smooth. So applying Lemma 3 to $\mathbf{v}^{\prime \prime}$ yields $-\mathbf{v}^{\prime \prime} \cdot \mathbf{d}<\mathbf{d}(n-1)+\mathbf{d}(n)$. This implies $\mathbf{v} \cdot \mathbf{d}>0$.

We now turn to the proof of Theorem 1. We should note that Lemma 4 is not necessary for this proof. The following argument works for any nonzero smooth vector $\mathbf{v}$. On the other hand, Lemma 4 indicates which nonzero smooth vectors might dot with $\mathbf{d}$ to give zero, namely smooth vectors $\mathbf{v}$ with $\mathbf{v}(\operatorname{dim}(\mathbf{v})) \in\{-1,1\}$. We now proceed by induction and use the technique outlined in the introduction.

Suppose we have shown that for any nonzero smooth vector $\mathbf{v}^{\prime}$ having dimension $m-1$ or less, $\mathbf{d} \cdot \mathbf{v}^{\prime} \neq 0$. Let $\mathbf{v}$ be a smooth nonzero $\mathrm{m}$-dimensional vector. Without loss of generality, $\mathbf{v}(m)>0$. We will inductively construct a sequence of $\mathrm{m}-$ dimensional vectors $\mathbf{w}_{m}, \mathbf{w}_{m-1}, \mathbf{w}_{m-2}, \ldots, \mathbf{w}_{2}$ that have the following properties for $i=m, \ldots, 2$ :

1. $\mathbf{w}_{i}(j)=\mathbf{v}(j)$ for $j \geq i$.

2. $\mathbf{w}_{i} \cdot \mathbf{d}=0$.

3 . $\mathbf{w}_{i}$ is not smooth. 
The first two properties will be immediate from the construction. The third property is what will require most of our work.

For $i \geq 2$ let $\mathbf{x}_{i}$ be the vector:

$$
\mathbf{x}_{i}(j)=\left\{\begin{aligned}
1 & \text { if } i=j \\
-1 & \text { if } i-\mathbf{b}(i) \leq j \leq i-1, \\
0 & \text { otherwise. }
\end{aligned}\right.
$$

Set $\rho_{m}=\mathbf{v}(m)$ (which is greater than zero) and $\mathbf{w}_{m}=\rho_{m} \mathbf{x}_{m}$. After $\mathbf{w}_{i+1}$ has been constructed, let $\rho_{i}=\mathbf{v}(i)-\mathbf{w}_{i+1}(i)$, and set $\mathbf{w}_{i}=\mathbf{w}_{i+1}+\rho_{i} \mathbf{x}_{i}$. Properties 1 and 2 are immediate.

We must now verify property 3 . This verification hinges on an analysis of the $\rho_{i}$ 's. The smoothness of $\mathbf{v}$ often forces the behavior of the $\rho_{i}$ 's. For example, $\mathbf{w}_{m}(m)-\mathbf{w}_{m}(m-1) \geq 2$ implies $\rho_{m-1}>0$. This is a pattern which continues through the first few $\rho_{i}$ 's.

Claim 1. For $m-\mathbf{b}(m) \leq i \leq m-1, \rho_{i}>0$.

Proof. Suppose inductively that $\rho_{i+1}, \ldots, \rho_{m}>0$. Then (using $i \geq m-\mathbf{b}(m)$ )

$$
\mathbf{w}_{i+1}(i)=-\sum_{j=i+1}^{m} \rho_{i} \leq-(m-i) .
$$

Since $\mathbf{v}(m) \geq 1$ and $\mathbf{v}$ is smooth, the smallest possible value of $\mathbf{v}(i)$ is $1-(m-i)$. So $\rho_{i}=\mathbf{v}(i)-\mathbf{w}_{i+1}(i) \geq 1-(m-i)+(m-i)=1$.

Claim 2. If $\rho_{t}, \ldots, \rho_{m}>0$ then $\mathbf{w}_{t}$ is not smooth.

Proof. When $m-\mathbf{b}(m)<t \leq m-1, \mathbf{w}_{t}$ fails to be smooth between coordinates $t-1$ and $t$. In this case $\mathbf{w}_{t}(t-1)=-\sum_{i=t}^{m} \rho_{i}$ while $\mathbf{w}_{t}(t)=\rho_{t}-\sum_{i=t+1}^{m} \rho_{i}$. This gives $\left|\mathbf{w}_{t}(t)-\mathbf{w}_{t}(t-1)\right|=2 \rho_{t}>1$.

Suppose $t \leq m-\mathbf{b}(m)$. We now verify the claim when $t$ is small. If $t=2$, then $m \geq 4$ and $\mathbf{w}_{2}(1)=-\rho_{2}-\rho_{3}$. If $t=3$, then $m \geq 6$ and $\mathbf{w}_{3}(1)=-\rho_{3}$ while $\mathbf{w}_{3}(2)=-\rho_{3}-\rho_{4}-\rho_{5}$. In either case $(t=2$ or $t=3) \mathbf{w}_{t}$ is not smooth. So we can assume that $t \geq 4$ and $t-\mathbf{b}(t)-1 \geq 1$.

Suppose $4 \leq t \leq m-\mathbf{b}(m)$. The interesting part of $\mathbf{w}_{t}$ is in coordinates $i$ where $t-\mathbf{b}(t) \leq i \leq t-1$, because these are the coordinates where $\mathbf{w}_{t}$ neither equals 0 nor necessarily agrees with $\mathbf{v}$. In particular, $\mathbf{w}_{t}$ might fail to be smooth around these indices (i.e. between two of these indices or between the first of these indices and the index preceding it or between the last of these indices and the index following it).

Set $\mathbf{c}(k)=\max \{n: n-\mathbf{b}(n) \leq k\}$. Then for $t-\mathbf{b}(t) \leq i \leq t-1$ we have

$$
\mathbf{w}_{t}(i)=-\sum_{j=t}^{\mathbf{c}(i)} \rho_{j} .
$$

Since $\mathbf{c}(k)$ is strictly increasing, $\mathbf{w}_{t}(t-\mathbf{b}(t)), \ldots, \mathbf{w}_{t}(t-2), \mathbf{w}_{t}(t-1)$ is a strictly decreasing sequence of negative numbers.

We will show that

(4) $\exists t-\mathbf{b}(t)-1 \leq s \leq t-2$ s.t. $\mathbf{c}(s+1)=\mathbf{c}(s)+2$ and $\mathbf{c}(s+1) \geq t+1$,

whence $\mathbf{w}_{t}(s+1) \leq \mathbf{w}_{t}(s)-2$ and $\mathbf{w}_{t}$ is not smooth. The existence of such an $s$ comes from the rule by which we constructed $\mathbf{b}$. Let $y=\mathbf{c}(t-1)$. Note that $y<m$. 
Since $y+1-\mathbf{b}(y+1)>t-1$ we have $\mathbf{b}(y+1)=\mathbf{b}(y)$. Applying (2) we get

$$
\mathbf{b}(t)=\mathbf{b}(y+1-\mathbf{b}(y+1))<\mathbf{b}(y+1)=\mathbf{b}(y) .
$$

It follows that there exists $u$ with $t \leq u<y$ and $\mathbf{b}(u+1)=\mathbf{b}(u)+1$. Then $s=u-\mathbf{b}(u)-1$ has the desired properties.

In the case where $\rho_{i}>0$ for all $i$ we've shown that property 3 holds. This is, of course, not always the case, but we will be able to show that in the other cases $\mathbf{v} \cdot \mathbf{d}$ cannot possibly be zero.

Claim 3. If there exists $i$ such that $\rho_{i} \leq 0$, then $\mathbf{v} \cdot \mathbf{d} \neq 0$.

Proof. Choose $t$ so that $\rho_{i}>0$ for all $i \geq t$ and $\rho_{t-1} \leq 0$. By Claim $1, t \leq m-\mathbf{b}(m)$. Clearly, $t \neq 2$. If $t=3$ then $m \geq 6, \mathbf{w}_{3}(2) \leq-\rho_{3}-\rho_{4}-\rho_{5}$, the smoothness of $\mathbf{v}$ implies $\mathbf{v}(2)>\mathbf{w}_{3}(2)$, and we have a contradiction. So $4 \leq t \leq m-\mathbf{b}(m)$.

Therefore, the description of $\mathbf{w}_{t}$ on the interval $t-\mathbf{b}(t) \leq i \leq t-1$ given by (3) and (4) holds. To reiterate, $\mathbf{w}_{t}(i)=0$ for $1 \leq i<t-\mathbf{b}(t)$, the sequence $\mathbf{w}_{t}(t-\mathbf{b}(t)), \ldots, \mathbf{w}_{t}(t-2), \mathbf{w}_{t}(t-1)$ is a strictly decreasing sequence of negative numbers, and there exists $t-\mathbf{b}(t)-1 \leq s<t-1$ such that $\mathbf{w}_{t}(s)-2 \geq \mathbf{w}_{t}(s+1)$.

Consider the vector $\mathbf{z}=\mathbf{v}-\mathbf{w}_{t}$. Property 2 of $\mathbf{w}_{t}$ implies $\mathbf{v} \cdot \mathbf{d}=\mathbf{z} \cdot \mathbf{d}$. Property 1 of $\mathbf{w}_{t}$ implies $\mathbf{z}(i)=0$ for $i \geq t$. Since we're assuming $\rho_{t-1} \leq 0$, we must have $\mathbf{w}_{t}(t-1) \geq \mathbf{v}(t-1)$. Since $\mathbf{w}_{t}(t-\mathbf{b}(t)-1), \mathbf{w}_{t}(t-\mathbf{b}(t)), \ldots, \mathbf{w}_{t}(t-2), \mathbf{w}_{t}(t-1)$ is strictly decreasing and $\mathbf{v}$ is smooth we must have $\mathbf{w}_{t}(i) \geq \mathbf{v}(i)$ for $t-\mathbf{b}(t)-1 \leq i \leq$ $t-1$. Furthermore, the existence of the double jump between $\mathbf{w}_{t}(s)$ and $\mathbf{w}_{t}(s+1)$ implies that $\mathbf{w}_{t}(i)>\mathbf{v}(i)$ for $t-\mathbf{b}(t)-1 \leq i \leq s$. This means that $\mathbf{z}(i) \leq 0$ for $t-\mathbf{b}(t)-1 \leq i \leq t-1$, and $\mathbf{z}(i)<0$ for $t-\mathbf{b}(t)-1 \leq i \leq s$. We know less about the values of $\mathbf{z}(i)$ for $i<t-\mathbf{b}(t)-1$. We know only that $\mathbf{z}$ is smooth on this interval. We now consider cases.

Case 1. $\mathbf{z}(i)<0$ for $i \leq s$. Clearly, $0>\mathbf{z} \cdot \mathbf{d}=\mathbf{v} \cdot \mathbf{d}$.

For the remaining two cases let $q=\max \{i<t-\mathbf{b}(t)-1: \mathbf{z}(i)=0\}$. When we restrict $\mathbf{z}$ to its first $q-1$ components (which are also the first $q-1$ components of v) we get a smooth vector $\mathbf{y}$ with $|\mathbf{y}(q-1)| \leq 1$.

Case 2. There exists $r>q+1$ such that $\mathbf{z}(r)<0$.

Applying Lemma 3 to the vector $\mathbf{y}$, we get

$$
0>\mathbf{y} \cdot \mathbf{d}-\mathbf{d}(q+1)-\mathbf{d}(q+2) \geq \mathbf{y} \cdot \mathbf{d}-\mathbf{d}(q+1)-\mathbf{d}(r) \geq \mathbf{z} \cdot \mathbf{d}=\mathbf{v} \cdot \mathbf{d} .
$$

Case 3. For all $r>q+1, \mathbf{z}(r)=0$.

We must have $s=q+1=t-\mathbf{b}(t)-1$. Since $\mathbf{v}$ is smooth and $\mathbf{z}(q)=\mathbf{v}(q)=0$, $\mathbf{z}(q+1)=\mathbf{v}(q+1)=-1$. Thus $\mathbf{z}$ is a smooth $(q+1)$-dimensional vector, and by our inductive hypothesis $\mathbf{v} \cdot \mathbf{d}=\mathbf{z} \cdot \mathbf{d} \neq 0$.

\section{Generalization I}

How can we modify the Conway-Guy construction and still get sets that have distinct subset sums? One natural approach is to change the sequence $\mathbf{b}$ while leaving steps 2 and 3 of the construction alone. Let's call a sequence $\mathbf{b}$ that by the above process generates a sequence of sets of integers that all have distinct subset sums a Conway-Guy generator (a $C G$-generator). What sequences b are CG-generators? 
Theorem 2. If $\boldsymbol{b}$ satisfies the following three properties then $\boldsymbol{b}$ is a CG-generator.

1. $\mathbf{b}(1)=0$.

2. $\mathbf{b}(i+1) \in\{\mathbf{b}(i), \mathbf{b}(i)+1\}$.

3. $\mathbf{b}(i-\mathbf{b}(i))<\mathbf{b}(i)$ for $i>1$.

The proof of Theorem 1 can be used (virtually word for word) to prove Theorem 2. While this is a list of sufficient conditions, a condition similar to 3 is actually necessary. In fact, if in a sequence $\mathbf{b}$ the integer $\mathrm{k}$ appear $k+1$ times in a row, then the sets produced will not have distinct subset sums. Say that $\mathbf{b}(i)=\mathbf{b}(i+1)=$ $\ldots=\mathbf{b}(i+k)=k$. Using the notation from above, set $\mathbf{v}=\sum_{j=i}^{k} \mathbf{x}_{j}$. Then $\mathbf{v}$ is smooth and $\mathbf{v} \cdot \mathbf{d}=0$. Condition 2 , on the other hand, is not necessary. Let $k$ be an integer greater than 3 . The vector $\mathbf{b}$ defined by

$$
\mathbf{b}(i)= \begin{cases}i-1 & \text { if } i \leq k, \\ {[\sqrt{2(i-k+2)}]} & \text { if } i>k,\end{cases}
$$

is a CG-generator (proof omitted). We don't see any necessary and sufficient conditions for a sequence to be a CG-generator.

\section{Generalization II}

In the mid 1980's Fred Lunnon conducted an extensive computational investigation of this problem. Among other things he determined $f(n)$ for $n \leq 8$ by exhaustive search $(f(n)$ is given by the Conway-Guy sequence in these cases) and verified that the Conway-Guy sequence has distinct subset sums for $n \leq 80$. He also found some sets that have distinct subset sums and beat the Conway-Guy sequence. These examples were in the form of four different sequences of sets. He verified that the first 67 sets from each of these sequences has distinct subset sums and conjectured that all sets in each of these sequences have distinct subset sums $[\mathrm{L}]$. These four sequences are given by $\mathbf{d}_{2,1}, \mathbf{d}_{2,2}, \mathbf{d}_{3,1}$, and $\mathbf{d}_{3,2}$ in the following construction.

We will describe these constructions by giving infinite dimensional difference vectors. Each one of these difference vectors corresponds to a sequence of sets. This correspondence is determined as in step 3 of the Conway-Guy construction above. In each of these sequences the first few differences will not be sums of previous differences; they will instead be given by a particular permutation of the set $\left\{1,1,2, \ldots, 2^{k}\right\}$ for an appropriate $k$ (as in the example given in the Introduction). The remaining differences will be the sums of previous differences.

Let $k$ be a positive integer. We define two slightly different difference vectors, $\mathbf{d}_{k, 1}$ and $\mathbf{d}_{k, 2}$. Again each sequence is defined by its own 'rule' vector $\mathbf{b}$, as follows,

$$
\begin{gathered}
\mathbf{b}_{k, 1}(2 k+1)=k+1, \quad \mathbf{b}_{k, 1}(2 k+2)=k+1, \quad \mathbf{b}_{k, 1}(2 k+3)=k+2, \\
\mathbf{b}_{k, 1}(2 k+i)=[\sqrt{2(i+2)}] \quad \text { for } \quad i \geq 4, \\
\mathbf{d}_{k, 1}(j)=\left\{\begin{aligned}
1 & \text { if } j=k, \\
4^{i-1} & \text { if } j=k+i \text { for } 1 \leq i \leq k, \\
2\left(4^{i-1}\right) & \text { if } j=k-i \text { for } 1 \leq i \leq k-1, \\
\sum_{l=j-\mathbf{b}_{k, 1}(j)}^{j-1} \mathbf{d}(l) & \text { if } j>2 k,
\end{aligned}\right.
\end{gathered}
$$

$\mathbf{b}_{k, 2}(2 k+2)=k+1, \quad \mathbf{b}_{k, 2}(2 k+3)=k+2, \quad \mathbf{b}_{k, 2}(2 k+4)=k+2$, 


$$
\begin{gathered}
\mathbf{b}_{k, 2}(2 k+i)=[\sqrt{2(i+1)}] \quad \text { for } i \geq 5, \\
\mathbf{d}_{k, 2}(j)=\left\{\begin{aligned}
1 & \text { if } j=k+1 \\
4^{i-1} & \text { if } j=k+1-i \text { for } 1 \leq i \leq k, \\
2\left(4^{i-1}\right) & \text { if } j=k+1+i \text { for } 1 \leq i \leq k, \\
\sum_{l=j-\mathbf{b}_{k, 2}(j)}^{j-1} \mathbf{d}(l) & \text { if } j>2 k+1 .
\end{aligned}\right.
\end{gathered}
$$

Thus, for example, we have

$$
\begin{gathered}
\mathbf{d}_{3,1}=(8,2,1,1,4,16,22,43,86,151,302, \ldots), \\
\mathbf{d}_{3,2}=(16,4,1,1,2,8,32,43,86,171,200,400, \ldots) .
\end{gathered}
$$

Note that $\mathbf{d}_{1,2}$ is just the difference vector from the Conway-Guy sequence.

Theorem 3. Let $n$ and $m$ be positive integers such that $m>2 n$. The following sets have distinct subset sums:

$$
S=\left\{\sum_{i=j}^{m} \mathbf{d}_{n, 1}(i): j=1, \ldots, m\right\}, \quad S^{\prime}=\left\{\sum_{i=j}^{m} \mathbf{d}_{n, 2}(i): j=1, \ldots, m\right\} .
$$

For $k \geq 2$ both of these difference vectors $\left(\mathbf{d}_{k, 1}\right.$ and $\left.\mathbf{d}_{k, 2}\right)$ give sequences of sets of integers that eventually beat the Conway-Guy sequence. But, the best upper bound we can get from these examples - approximately $f(n)<.22002\left(2^{n}\right)$ - is only slightly better than the previous best known upper bound - approximately $f(n)<.2246\left(2^{n}\right)$ - which was given by the 67 th set corresponding to the vector $\mathbf{d}_{3,2}$. These are the only constructions I know of that beat (or even have a chance of beating) the Conway-Guy construction. For a more complete discussion of these examples and a proof of Theorem 3, see [B].

\section{Conclusion}

There are several questions that remain wide open. The most notable of these are the original conjecture of Erdös and necessary and sufficient conditions for a sequence to be a CG-generator. Here we mention one more question motivated by the above results.

We begin with some motivation. Suppose $S=\left\{a_{1}<a_{2}<\ldots<a_{n}\right\}$ has distinct subset sums and $a_{n} \leq c 2^{n}$ for some $c$ (not necessarily a constant). Set $\mathbf{a}=\mathbf{a}_{S}=\left(a_{1}, a_{2}, \ldots, a_{n}\right)$. For ease of notation set $L_{1}:=\{-1,0,1\}^{n}$, set $L_{2}:=$ $\{-2,-1,0,1,2\}^{n} \backslash L_{1}$ and write $H_{S}$ for the hyperplane $\left\{\mathbf{u} \in \mathbf{R}^{n}: \mathbf{u} \cdot \mathbf{a}=0\right\}$. We will show that

$$
a_{n} \leq c 2^{n} \Rightarrow\left|H_{S} \cap L_{2}\right| \geq 3^{n} /\left(c n 2^{n+1}\right) .
$$

For every vector $\mathbf{u} \in L_{1}$ the bound on the size of elements of $S$ implies $-c n 2^{n}<$ $\mathbf{u} \cdot \mathbf{a}<c n 2^{n}$. So for some $k$ the set $\left\{\mathbf{u} \in L_{1}: \mathbf{u} \cdot \mathbf{a}=k\right\}$ contains at least $3^{n} /\left(c n 2^{n+1}\right)$ elements (I believe a much greater concentration must occur, but I have no proof of this). Let $\left\{\mathbf{u} \in L_{1}: \mathbf{u} \cdot \mathbf{a}=k\right\}=\left\{\mathbf{u}_{1}, \mathbf{u}_{2}, \ldots\right\}$. Since $\mathbf{u}_{1} \cdot \mathbf{a}=k$ and $\mathbf{u}_{2} \cdot \mathbf{a}=k$, $\left(\mathbf{u}_{1}-\mathbf{u}_{2}\right) \cdot \mathbf{a}=0$. Since $S$ has distinct subset sums, $\mathbf{u}_{1}-\mathbf{u}_{2} \in L_{2}$. Repeating this argument for all pairs of the form $\left(\mathbf{u}_{1}, \mathbf{u}_{j}\right)$, it follows that the set $H_{S} \cap L_{2}$ must contain at least $3^{n} /\left(c n 2^{n+1}\right)$ elements (I believe this cardinality must be much larger, but, again, I have no proof).

So there appears to be an inverse relationship between the size of the largest element of $S$ and the cardinality of $H_{S} \cap L_{2}$. In order to find a set of integers with distinct subset sums and small greatest element it might be a good idea to search 
for a hyperplane whose intersection with $L_{1}$ is the zero vector (this is required for the distinct subset sums condition) and whose intersection with $L_{2}$ is large.

How big is large? Among the examples discussed in this paper the cardinality of $H_{S} \cap L_{2}$ appears to be largest when $S=\left\{1,2, \ldots, 2^{n-1}\right\}$. In order to calculate the cardinality in this case set

$$
\mathbf{e}_{i}(j)= \begin{cases}2 & \text { if } j=i, \\ 1 & \text { if } j=i+1, \\ 0 & \text { otherwise. }\end{cases}
$$

The set $\left\{\mathbf{e}_{1}, \ldots, \mathbf{e}_{n-1}\right\}$ is a basis for $H_{S}$. The linear combination $c_{1} \mathbf{e}_{1}+c_{2} \mathbf{e}_{2}+$ $\ldots+c_{n-1} \mathbf{e}_{n-1}$ is in $L_{2} \cup\{\mathbf{0}\}$ if and only if $c_{i} \in\{-1,0,1\}$ for all $i$ and $c_{i} c_{i+1} \neq 1$ for all $i$. Let $f_{n}$ be the number of such linear combinations. Using the recurrence relation $f_{n}=2 f_{n-1}+f_{n-2}$, we see that $f_{n}=1 / 2(1+\sqrt{2})^{n}+1 / 2(1-\sqrt{2})^{n}$. Is this the optimal example?

Conjecture 1. If $H$ is a hyperplane in $\mathbf{R}^{n}$ and $H \cap\{-1,0,1\}^{n}=\{\mathbf{0}\}$, then

$$
\left|H \cap\{-2,-1,0,1,2\}^{n}\right| \leq 1 / 2(1+\sqrt{2})^{n}+1 / 2(1-\sqrt{2})^{n} .
$$

Since the connection between $\max S$ and the cardinality of $H_{S} \cap L_{2}$ given in (5) is so weak a proof of this conjecture would say little about the conjecture of Erdős. On the other hand, a counterexample to this conjecture might suggest how a counterexample to the conjecture of Erdős could be found.

\section{REFERENCES}

[AS] N. Alon and J. Spencer, The Probabilistic Method, Wiley, New York, 1992. MR 93h:62002 [B] T. Bohman, A Construction For Sets of Integers With Distinct Subset Sums, in preparation.

[CG] J.H. Conway and R.K. Guy, Sets of natural numbers with distinct sums, Notices Amer. Math. Soc. 15(1968), 345.

[E1] P. Erdős, personal communication.

[E2] P. Erdős, Problems and results from additive number theory, Colloq. Théorie des Nombres, Bruxelles, 1955, Liège \& Paris, 1956, 127-137, esp. p. 137. MR 18:18a

[G1] R. K. Guy, Sets of integers whose subsets have distinct sums, Theory and Practice of Combinatorics (A. Kotzig 60th Birthday Vol.), Ann. of Discrete Math., vol. 12, North-Holland, Amsterdam, 1982, pp. 141-154. MR 86m:11009

[G2] R. K. Guy, Unsolved Problems in Number Theory, 2nd ed., Springer-Verlag, New York, 1994. CMP 95:02

[L] W. F. Lunnon, Integer sets with distinct subset sums, Math. Comp., 50(1988) 297-320. MR 89a:11019

Department of Mathematics, Rutgers University, New Brunswick, New Jersey 08903

E-mail address: bohman@math.rutgers.edu

Current address: Department of Mathematics, Massachusetts Institute of Technology, Cambridge, Massachusetts 02139 